\title{
Effects of boundary condition and cell structure on dynamic axial crushing honeycomb
}

\author{
Tsutomu UMEDA* and Koji MIMURA* \\ * Division of Mechanical Engineering, Graduate School of Engineering, Osaka Prefecture University \\ 1-1, Gakuen-cho, Naka-ku, Sakai, Osaka 599-8531, Japan \\ E-mail: umeda@me.osakafu-u.ac.jp
}

Received: 1 November 2017; Revised: 14 March 2018; Accepted: 9 April 2018

\begin{abstract}
The dynamic axially crushing behavior of metal honeycombs was studied with placing emphasis on the effects of boundary condition and cell structure on its characteristics as an energy absorber. Numerical honeycomb models of some metal foil materials were made by taking the plastic deformation of adhesive layer, the failure of adhesivelybonded joint and the initial imperfection into account. Then, firstly, the foil material and the branch angle $\varphi$ of cell geometry were varied to examine the effects on the crushing behavior and the energy absorption capacity. Secondly, to investigate the effects of boundary condition on the crushing mode and the energy absorption capacity, some boundary conditions such as the fixed end condition, the contact condition at the upper and lower ends and the periodic boundary condition at the side surfaces were applied to the honeycomb model. Typical calculated results under different strain rates and geometric conditions were compared with the corresponding experimental results, and the effects of material properties on the mean buckling stress were discussed. It was found that for the material with strain rate dependence, the stress increment of crushing honeycomb due to increasing the strain rate was 30$40 \%$ as large as that of the material itself. Small $\varphi$ causes different deformation mode of wrinkles, and the mean buckling stress $\sigma_{m}$ decreased about $50 \%$ by changing $\varphi$ from $120^{\circ}$ to $30^{\circ}$. Furthermore, small-scale honeycomb with small $\varphi$ is easy to be affected by free edges considerably at $\varphi=60^{\circ}$. The oblique loading condition caused the transition from axial collapse to bending collapse so that at the crush angle of $45^{\circ} \sigma_{m}$ became $30-50 \%$ smaller than that under the vertical loading condition, while the effect of the direction of oblique load on $\sigma_{m}$ is not so large.
\end{abstract}

Keywords : Honeycomb, Energy absorption, Boundary condition, Strain rate dependence

\section{Introduction}

The metal honeycomb structure crushes with almost constant load during relatively long stroke in the longitudinal direction of cells so that it is a desirable energy absorber. In general, the honeycomb structure has various geometric patterns and has been extensively studied as a 2-dimensional cellular structure about its various macroscopic properties (Gibson and Ashby, 1997). For practical use, the hexagonal honeycomb structure of the highest specific strength is the most important. Commercial metal honeycombs are mostly made of aluminum foil sheets by adhesive bonding and expansion process, and many investigations on the energy absorption characteristic or the dynamic crushing behavior of metal honeycombs under out-of-plane compressive loading condition have been conducted (Wierzbicki, 1983, Wu and Jiang, 1997, Ogasawara et al., 1997, Baker et al., 1998, Zhao and Gary, 1998, Yasui, 2000). As for the energy-absorbing mechanism of the metal honeycomb, Wierzbicki (1983) studied the mathematical model of its progressive buckling, which was based on the rigid-perfectly plastic material approximation and the energy considerations, and found simple formulas that finally gave the mean buckling stress as a function of the ratio of wall thickness to cell size. Ogasawara et al. (1997) also investigated this problem mainly by conducting a series of numerical analyses under various geometric conditions using the explicit FEM code LS-DYNA and obtained similar formula of the mean buckling stress by the method of least squares. Yamashita et al. (2005) performed experimental and numerical study to clarify the effect of branch angle and 
cell wall thickness on the crush behavior. Numerical analyses were mainly carried out for an unit cell model using the explicit FEM code DYNA3D, while the material model was a simple bilinear approximation, and the adhesively-bonded joint was not taken into consideration. Chen et al. (2007) especially studied the deformation behavior around three-fold point corner by the numerical analysis using the non-linear FEM code MSC.Marc to understand the energy absorption performance in axial compression and discussed the extension of Wierzbicki's model to be able to treat the seamless honeycomb without adhesive bonding. In our previous study (Umeda et al., 2011), Wierzbicki's model was extended to be able to represent the work hardening and the variation of geometry except the branch angle, and calculated results using the seamless honeycomb model of $6 \times 6$ cells, which was made in one united body for simplicity so that the effect of adhesively-bonded joint on the crush analysis was disregarded, roughly agreed with the respective predictions by the mathematical model.

In this study, the effect of adhesively-bonded joint between metal foil sheets was also considered. Some metal honeycombs were numerically modeled with taking the plastic deformation of adhesive layer, the fracture of adhesively-bonded joint and the initial imperfection into consideration. Then, axial crushing analyses were conducted by LS-DYNA (LSTC and JSOL, 2007) under low-speed and impact loading conditions that were the same conditions with the experiments conducted by two kinds of testing machines respectively. The branch angle in the hexagonal cell geometry was also varied from the regular value $120^{\circ}$ to 30,60 , or $180^{\circ}$. The contact condition including the embedded-process-zone (EPZ) model (Tvergaard and Hutchinson, 1993, Yang and Thouless, 2001), which assumes the linear traction-separation law for mixed mode interface fracture, was used. EPZ model reduces the load amplitude at the plateau part of load-displacement relationship to some extent and reproduces natural crushing behavior (Umeda et al., 2014). The initial imperfection due to the manufacturing process and the randomicity also greatly affects the crushing behavior, so that was also introduced into the numerical model. The calculated results obtained using this elaborated numerical model agreed very well with the experimental results for regular honeycombs of different materials. Then, the strain rate dependence of honeycomb structure was discussed as compared with that of the material itself. Especially, the effects of branch angle and boundary conditions on the crushing behavior and the energy absorption capacity were investigated. Furthermore, to clarify the availability in the actual situation of collision accident, the effects of oblique loading condition were also examined.

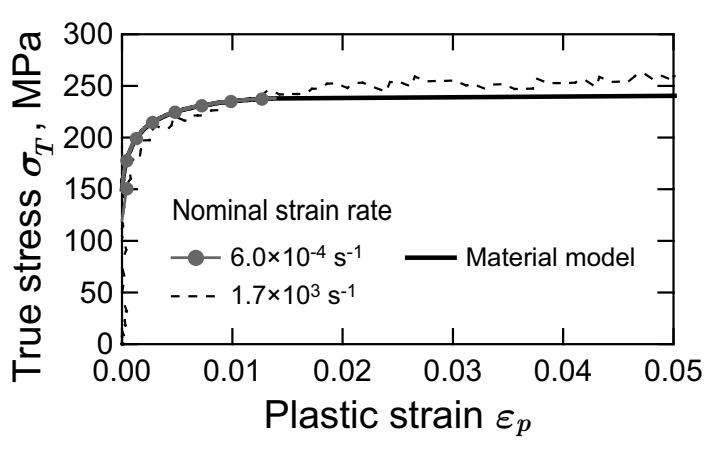

(a) A3003 foil

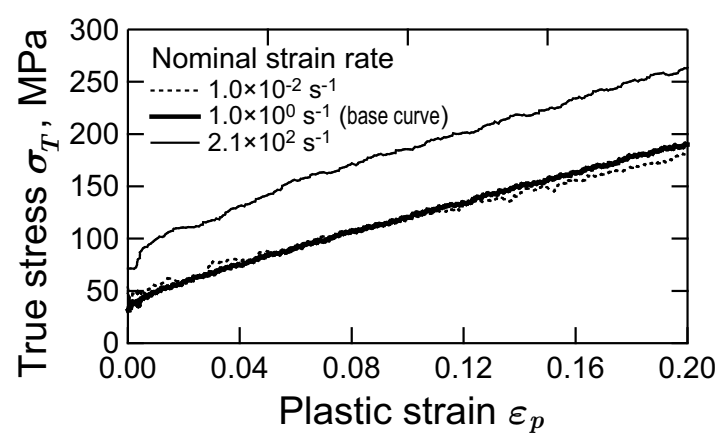

(c) Annealed C1100 foil

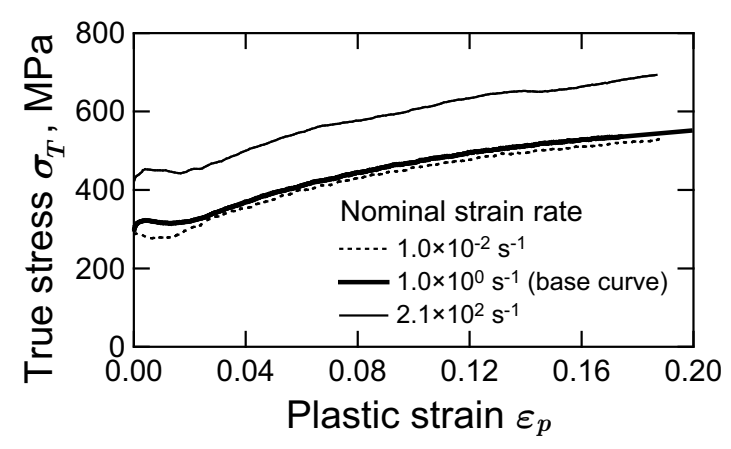

(b) Annealed SUS430 foil

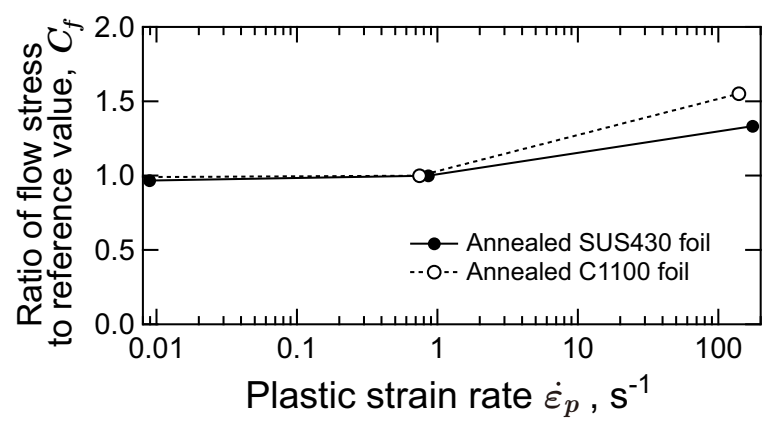

(d) Ratio of flow stress to reference value, $C_{f}\left(\dot{\varepsilon_{p}}\right)$

Fig. 1 True stress - plastic strain relationships were obtained by tension tests within a wide range of strain rate for three foil materials of (a) A3003 aluminum alloy, (b) annealed SUS430 stainless steel and (c) annealed C1100 tough pitch copper. The bold solid lines in those figures show (a) material model and (b)-(c) base curves used in the numerical simulation. 


\section{Foil Materials}

In the experiments, honeycomb cores were made of foil sheets of SUS430 stainless steel (annealed at $800{ }^{\circ} \mathrm{C}$ for 45 minutes and cooled in the vacuum of space), C1100 tough pitch copper (annealed at $425^{\circ} \mathrm{C}$ for 30 minutes " ) and A3003 aluminum alloy. Those were modeled as isotropic elastic/plastic materials based on the von Mises yield criterion. The flow stress $\left(\sigma_{T}\right)$ - plastic strain $\left(\varepsilon_{p}\right)$ relationships were given by the piecewise linear interpolation of the experimental data. Figures 1 (a)-(c) show the respective $\sigma_{T}-\varepsilon_{p}$ relationships obtained by the tension tests. The foil is so thin (the thickness, $h$, is $0.1 \mathrm{~mm}$ for SUS430 and C1100 foil and is $0.076 \mathrm{~mm}$ for A3003 foil) that the output voltage of load for only one sheet is too small to get accurate data. Therefore, the specimen was made of 10 sheets of foil with gluing together at the grip parts (Umeda, et al., 2011). In Fig. 1 (a), the lines for the quasi-static (the nominal strain rate $6.0 \times 10^{-4} \mathrm{~s}^{-1}$ ) and the high strain rate $\left(1.7 \times 10^{3} \mathrm{~s}^{-1}\right)$ tension tests agree well with each other except very small failure strain in the quasi-static tension. The bold line shows the approximation of $\sigma_{T}-\varepsilon_{p}$ relationship used in the numerical simulation. In Figs. 1 (b) and (c), $\sigma_{T}$ increases with the plastic strain rate $\dot{\varepsilon_{p}}$, and the data at $\dot{\varepsilon_{p}} \cong 1.0 \times 10^{0} \mathrm{~s}^{-1}$ was used as the base curve in the calculation with the piecewise linear interpolation and extrapolation. The flow stress was evaluated by scaling corresponding reference value, which was obtained at calculated $\varepsilon_{p}$ from the base curve, with the ratio of current flow stress to its reference value, $C_{f}\left(\dot{\varepsilon_{p}}\right) . C_{f}\left(\dot{\varepsilon_{p}}\right)$ was determined from the experimental data as shown in Fig. 1 (d) and was evaluated in the numerical simulation for calculated $\dot{\varepsilon_{p}}$ using piecewise linear interpolation and extrapolation.

Table 1 Dimensions of honeycomb core models $\left(6 \times 6\right.$ cells, $\left.h^{\prime}=2 h\right)$.

\begin{tabular}{c|c|c|c|c|c}
\hline Materials & $D(\mathrm{~mm})$ & $D^{\prime}(\mathrm{mm})$ & $h(\mathrm{~mm})$ & $\varphi\left(^{\circ}\right)$ & $L(\mathrm{~mm})$ \\
\hline SUS430, C1100 & 3.67 & 3.67 & 0.1 & 120 & 25 \\
\hline A3003 & $"$ & $"$ & 0.076 & $120,30,60,180$ & $"$ \\
\hline
\end{tabular}

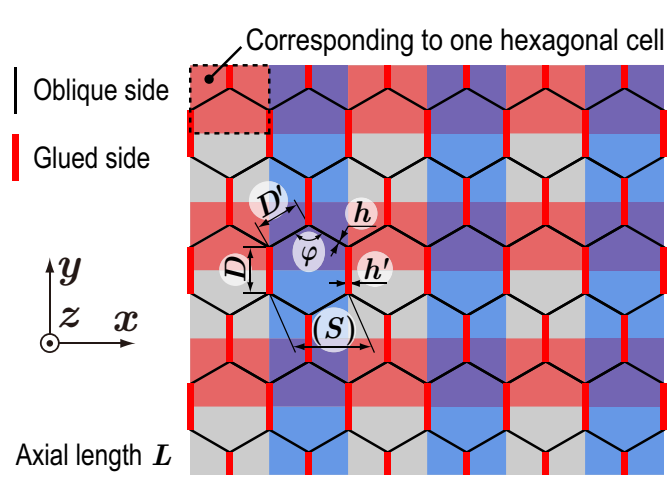

(a) Schematic of cell geometry

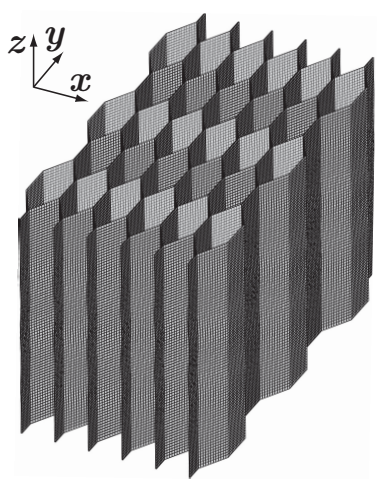

(c) $\varphi=60^{\circ}$

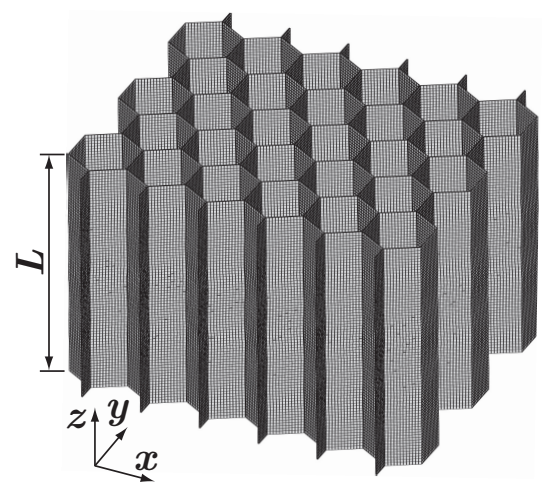

(b) $\varphi=120^{\circ}$

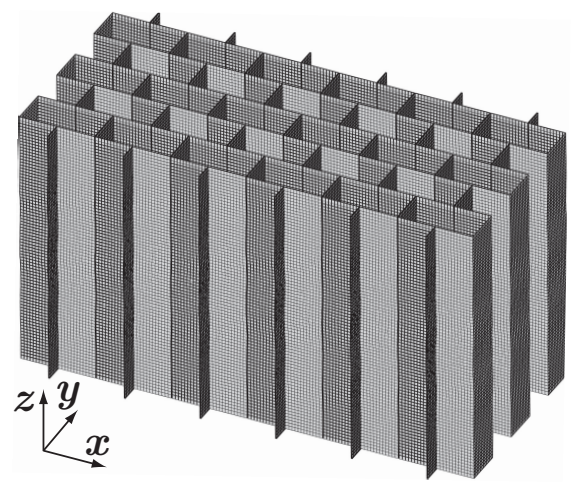

(d) $\varphi=180^{\circ}$

Fig. 2 Honeycomb core models. (a) shows the definitions of dimensions in the cell geometry of $6 \times 6$ cells hoenycomb, and (b)-(d) show finite element models of $\varphi=120,60$ and $180^{\circ}$ respectively.

\section{Numerical model}

\subsection{FE model and boundary conditions}

Figure 2 (a) shows the schematic view of $6 \times 6$ cells honeycomb core in the axial direction. The cell geometry is 
defined with the glued side length $(D)$, the oblique side length $\left(D^{\prime}\right)$, the branch angle $(\varphi)$, and the axial length $(L)$ besides the foil thickness $(h)$ and the thickness of glued side $\left(h^{\prime}\right)$. Figures 2 (b)-(d) show finite element models of $\varphi=120,60$ and $180^{\circ}$ respectively. In this study, to evaluate the effect of branch angle, the number of cells on a side is fixed to be six, $D=D^{\prime}=3.67 \mathrm{~mm}, h^{\prime}=2 h$ and $L=25 \mathrm{~mm}$ (see Table 1). Figure 3 (a) shows a typical finite element model $(\varphi=$ $\left.120^{\circ}\right)$ and the boundary conditions, which are common to all the models. The model consists of 139970 shell elements of Belytschko-Leviathan type (LSTC and JSOL, 2007) and 142688 nodes. The honeycomb core was set on the bottom rigid wall, which was fully fixed, and was crushed by the top rigid wall, which moved in the direction of the applied velocity $V$. For the dynamic crushing analysis, $V=3.4 \mathrm{~m} / \mathrm{s}$. Here, the rigid wall is defined as a non-deformable surface, and the nodes on that are not fixed and can move on the honeycomb core side of that. On the other hand, the honeycomb structure is often used to constitute a sandwich panel with two face plates that are glued to the upper and lower ends of honeycomb core using the adhesive. This situation will be also treated, and in this case, the nodes at the lower end are fully fixed for the translation and rotation in all directions, while those at the upper end are allowed to move in only the direction of applied velocity. At first, the normal impact loading condition was examined by applying $V$ in $-z$ direction. To reproduce the crushing behavior of honeycomb in the numerical simulation, the contact conditions with friction among neighboring foil sheets are needed. The coefficient of friction $\mu$ for those contact conditions was varied from the static coefficient $\mu_{s}=0.28$ to the kinetic coefficient $\mu_{k}=0.20$ continuously using the exponential coefficient $\beta=0.5 \mathrm{~s} / \mathrm{m}$ (LSTC and JSOL, 2007) as follows:

$$
\mu=\mu_{k}+\left(\mu_{s}-\mu_{k}\right) e^{-\beta\left|v_{r e l}\right|},
$$

where $v_{\text {rel }}$ means the relative velocity $(\mathrm{m} / \mathrm{s})$ between contact surfaces. To consider the plastic deformation of adhesive layer and the fracture of adhesively-bonded joint, EPZ model was also introduced to the contact condition as mentioned in subsection 3.3. The calculation of low-speed crushing under the normal loading condition was conducted both by reducing $V$ within the limits of computing capability and by equating the effect of strain rate on the stress-strain relationship in the calculation with that under actual strain rate condition. Here, $\dot{\varepsilon_{N}}$ shows the nominal strain rate. If the applied velocity in the calculation is $V^{c a l}$, the calculated nominal strain rate $\dot{\varepsilon_{N}^{c a l}}$ is evaluated as $V^{c a l} / L$ and $\dot{\varepsilon_{N}} / \dot{\varepsilon_{N}^{c a l}}=V / V^{c a l}$, so that $C_{f}\left(\dot{\varepsilon_{p}}\right)$ can be evaluated as follows:

$$
C_{f}\left(\dot{\varepsilon_{p}}\right) \cong C_{f}\left(\dot{\varepsilon_{p}^{c a l}} \times \frac{\dot{\varepsilon_{N}}}{\dot{\varepsilon_{N}^{c} a l}}\right)=C_{f}\left(\dot{\varepsilon_{p}^{c a l}} \times \frac{V}{V^{c a l}}\right),
$$

where $\dot{\varepsilon}_{p}^{c a l}$ is the calculated plastic strain rate. In the input file of LS-DYNA, $C_{f}\left(\dot{\varepsilon_{p}}\right)$ is given in the tabular form with the corresponding plastic strain rate. To equating the effect of strain rate, the data of corresponding plastic strain rate is multiplied by $V / V^{c a l}$.

The two boundary conditions at the upper and lower ends of model, which are mentioned above, correspond to the experimental conditions respectively. For the former condition, the contact surfaces between honeycomb core and rigid wall were assumed to have the friction coefficient of 0.28 , and the condition is referred to as "without face plates" in the

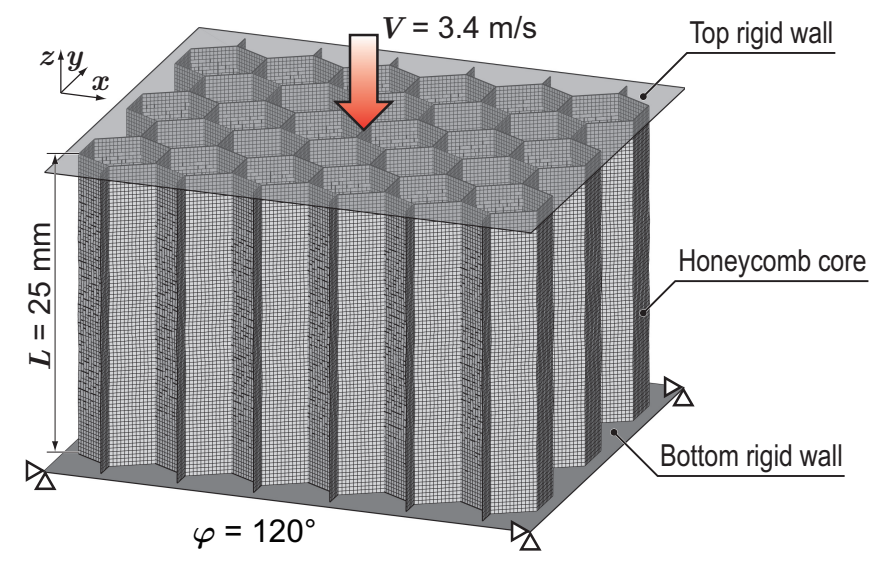

(a) Finite element model and basic boundary conditions

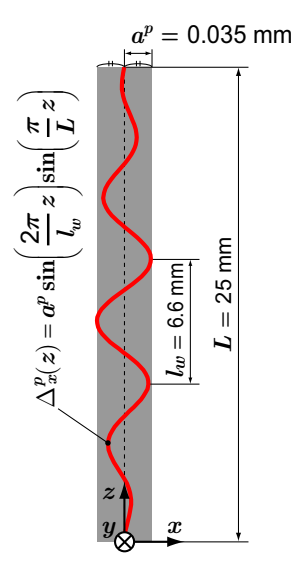

(b) Perturbation due to processing

Fig. 3 The numerical simulations were carried out using (a) the finite element model of honeycomb sandwich panel with (b) the perturbation that was modeled as the initial imperfection due to manufacturing process and was added to the coordinates of nodes $\left(\varphi=120^{\circ}\right.$, impact loading condition). 
following. For the latter condition, the nodes at the upper and lower ends were fully fixed on respective rigid surfaces, and that is referred to as "with face plates". Furthermore, the calculations under "the periodic boundary condition" in $x$ and $y$ directions were also carried out to examine the effect of free edges. There is a possibility that the crushing behavior under normal loading condition changes with $L$ if it is too small as compared with the wave length of wrinkle or the edge effect is significant. The edge effect at upper and lower ends is related to the occurrence of localized deformation and is precipitated for small $L$. In this study, $L$ is fixed to be $25 \mathrm{~mm}$ and is large enough as compared with the wave length of wrinkle. Then, only the edge effect at side surfaces, which is caused by the deformation of free surfaces at sides due to the lateral force component and becomes large if the ratio of the number of foil sheets at sides to all those in the honeycomb, is discussed to examine the validity of uniaxial condition or in-plane periodicity.

\subsection{Initial imperfection}

In this study, two types of perturbations were assumed. $\Delta^{p}(z)$ describes the infinitesimal deformation due to the manufacturing process such as rolling, expansion and so on. It greatly affects to reproduce the localized deformation observed in the experiment. The amplitude $a^{p}$ was assumed to be $0.035 \mathrm{~mm}$. The length $l_{w}=6.6 \mathrm{~mm}$ was determined based on the observation of the buckling pattern. Adhesively-bonded foil sheets are expanded in $x$ direction so that $\Delta^{p}(z)$ was assumed to have $x$ component, as shown in Fig. 3 (b).

$$
\Delta^{p}(z)=\left(\Delta_{x}^{p}(z), 0,0\right), \quad \Delta_{x}^{p}(z)=a^{p} \sin \left(\frac{2 \pi}{l_{w}} z\right) \sin \left(\frac{\pi}{L} z\right)
$$

Another perturbation $\Delta^{r}(z)$ introduces the randomicity in the dimensions and was also assumed to change the magnitude in $z$ direction. Here, $n_{g}$ is the number of nodes, and $\Delta(i)$ is the pseudo-random number obtained by the linear congruential method within $|\Delta(i)|<1$, and the amplitude $a^{r}$ was assumed to be $0.015 \mathrm{~mm}$. Then, the magnitude of the resultant perturbation $\Delta^{\text {tot }}(z)$ is less than $0.05 \mathrm{~mm}$ in $x, y$ and $z$ directions.

$$
\begin{aligned}
& \Delta^{r}(z)=\left(\Delta^{r}(3 n+1, z), \Delta^{r}(3 n+2, z), \Delta^{r}(3 n+3, z)\right) \quad\left(n=0, \ldots, n_{g}-1\right), \\
& \quad \Delta^{r}(i, z)=a^{r} \Delta(i) \sin \left(\frac{\pi}{L} z\right) \quad\left(i=1, \ldots, 3 n_{g}\right), \\
& \Delta^{t o t}(z)=\Delta^{p}(z)+\Delta^{r}(z) .
\end{aligned}
$$

\subsection{EPZ model}

The simplified linear traction-separation law (see Fig. 4) was used to represent the mixed mode fracture of adhesiveadherend interfaces. $\sigma^{*}$ and $\tau^{*}$ are the normal and shear stresses acting on the interface in the fracture process zone, and the fracture process starts when the condition:

$$
\left(\left\langle\sigma^{*}\right\rangle / \sigma^{c}\right)^{2}+\left(\tau^{*} / \tau^{c}\right)^{2}=1
$$

is satisfied (LSTC and JSOL, 2007). $\hat{\sigma}(0)=\sigma^{c}$ means the pure normal separation (mode I), and $\hat{\sigma}(0)=\tau^{c}(=35 \mathrm{MPa})$ means the pure shear separation (mode II) (Yang and Thouless, 2001). For the crack opening deformation, $\sigma^{*}=\tau^{*}=0$ if once the separation $\delta$ reached its critical value $\delta^{c}$.

\section{Calculated results}

In Figs. 5 (a)-(c), typical snapshots of deformed honeycomb core at the time $t \cong 50 \mu \mathrm{s}, 150 \mu \mathrm{s}$ and $4.55 \mathrm{~ms}$ are shown. The infinitesimal deformation of core at $t \cong 50 \mu \mathrm{s}$ in Fig. 5 (a) shows the buckling pattern at side surfaces. The contour plot shows the distribution of the equivalent stress. Figure 5 (b) shows the localized deformation of core at $t \cong 150 \mu \mathrm{s}$. Then, wrinkles develop from this part to the upper or lower part as shown in Fig. 5 (c). The localized

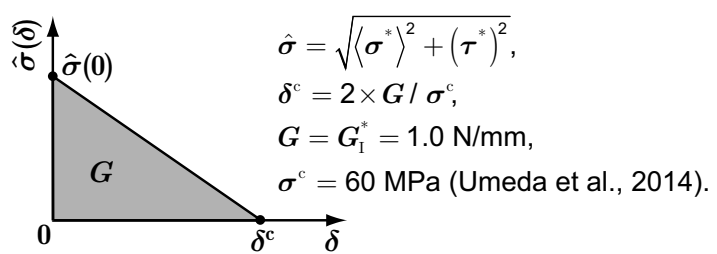

Fig. 4 Simplified linear traction-separation law is used to determine $\sigma^{*}$ and $\tau^{*}$ by linearly decreasing $\hat{\sigma}$ with $\delta$. 


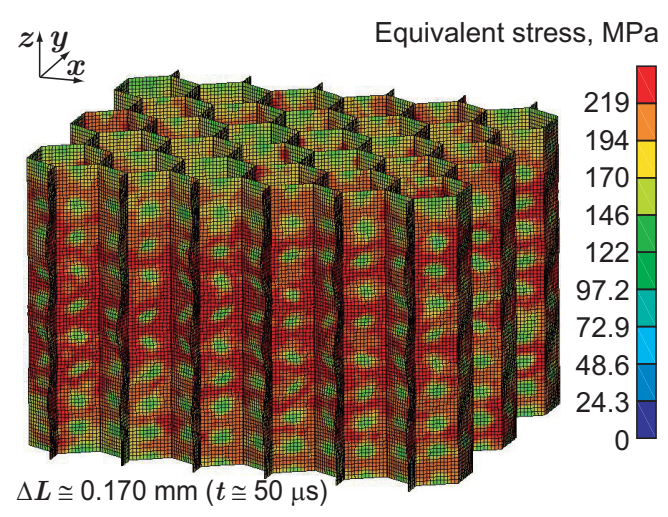

(a) Buckling pattern

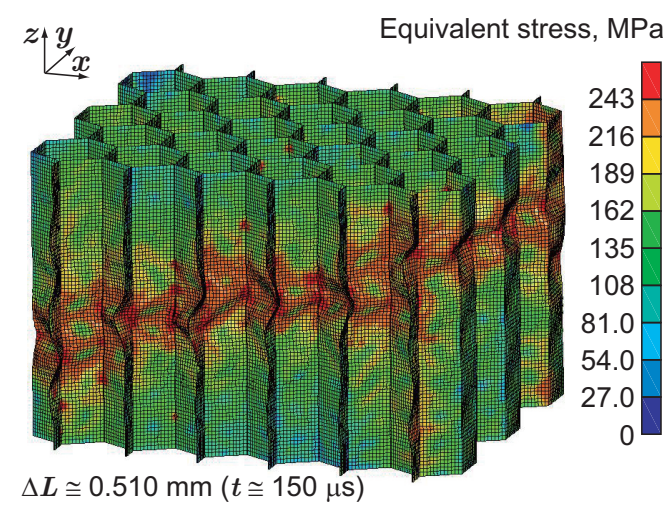

(b) Localized deformation

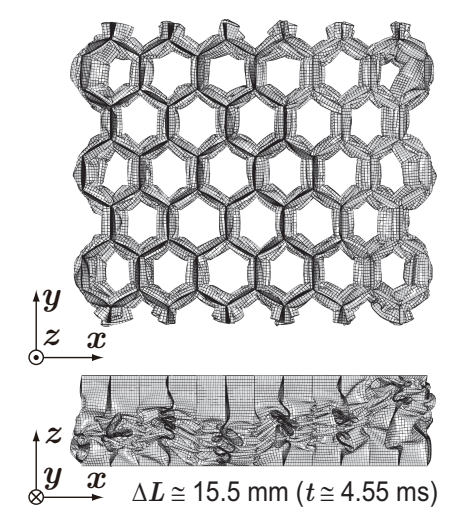

(c) Crushed core

Fig. 5 Calculated deformation of honeycomb core model shows (a) the buckling pattern, (b) the localization and (c) the progressive plastic buckling (A3003, with face plates, $\varphi=120^{\circ}$ ).

deformation observed in the experiment is reproduced by introducing the initial imperfection. The honeycomb model without initial imperfection successively yields wrinkles from the upper or lower end. In Fig. 6 (a)-(c), the crushed cores of $\varphi=30,60$ and $180^{\circ}$ at $t \cong 4.55 \mathrm{~ms}$ are shown respectively. The deformation mode of wrinkles for $\varphi=30$ and $60^{\circ}$ is different from that for $\varphi=120$ and $180^{\circ}$, and it seems that the free edges and structural anisotropy of the former greatly affect the crushing behavior as compared with that of the latter.

The energy absorption capacity of the honeycomb model was evaluated with using the mean buckling load $P_{m}$ and the mean buckling stress $\sigma_{m}$. Here, $P_{m}$ was defined as the area under axial load $\left(P\right.$ or $\left.P_{z}\right)$-displacement $\left(\Delta L\right.$ or $\left.\Delta L_{z}\right)$ curve dividing by the total displacement before densification, which was taken to be $12.5 \mathrm{~mm}$ in this paper (for example, see Figs. 7 (a) and (b)). $\sigma_{m}$ was defined as the area under nominal stress $\left(\sigma_{N}\right)$-apparent nominal strain $\left(\varepsilon_{N}\right)$ curve dividing by the total apparent nominal strain before densification, on the condition that $\sigma_{N}$ was evaluated as the axial load per the net area (except for hollow parts) of cross section to be able to compare with the strength of foil material, while many pieces

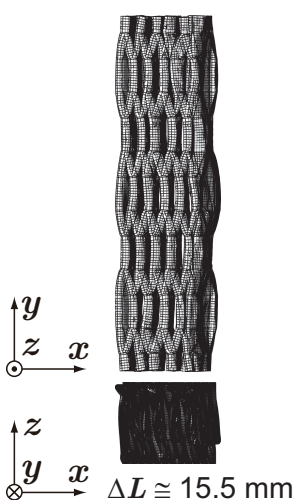

(a) $\varphi=30^{\circ}$

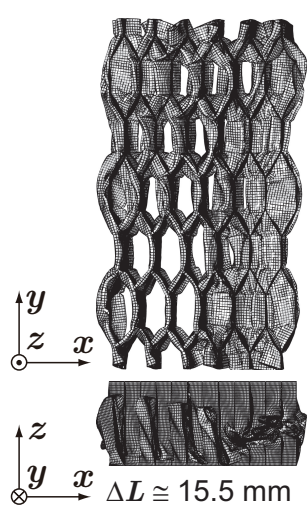

(b) $\varphi=60^{\circ}$

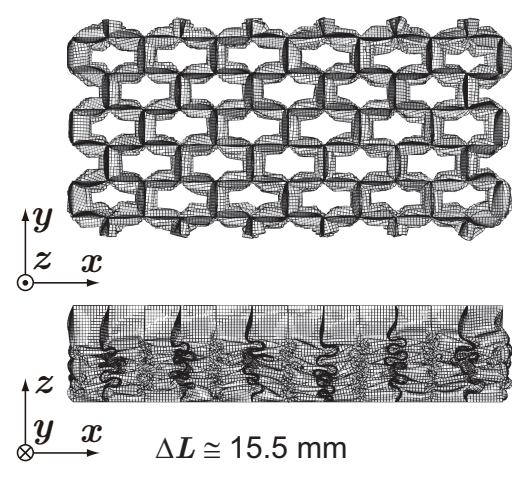

(c) $\varphi=180^{\circ}$

Fig. 6 Crushed irregular honeycomb core models of $\varphi=30^{\circ}, 60^{\circ}$ and $180^{\circ}$ (A3003, with face plates, $V=3.4$ $\mathrm{m} / \mathrm{s}, t \cong 4.55 \mathrm{~ms}$ ). 


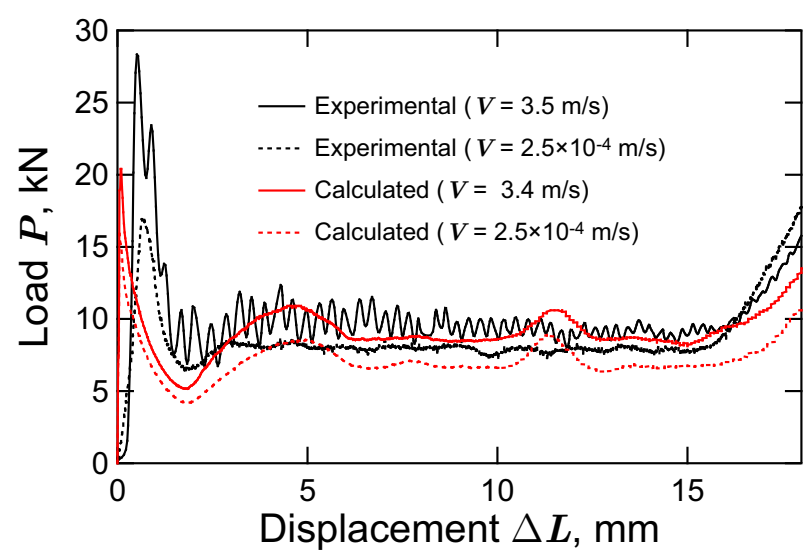

(a) Annealed SUS430 honeycomb

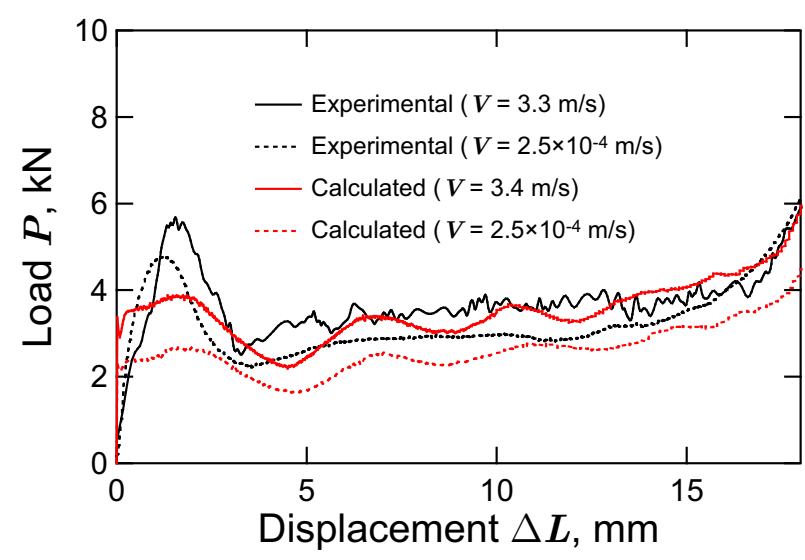

(b) Annealed C1100 honeycomb

Fig. 7 Load-displacement relationships of annealed SUS430 and C1100 were obtained under low-speed and impact loading conditions both by the experiment and by the numerical simulation $\left(\varphi=120^{\circ}\right)$.

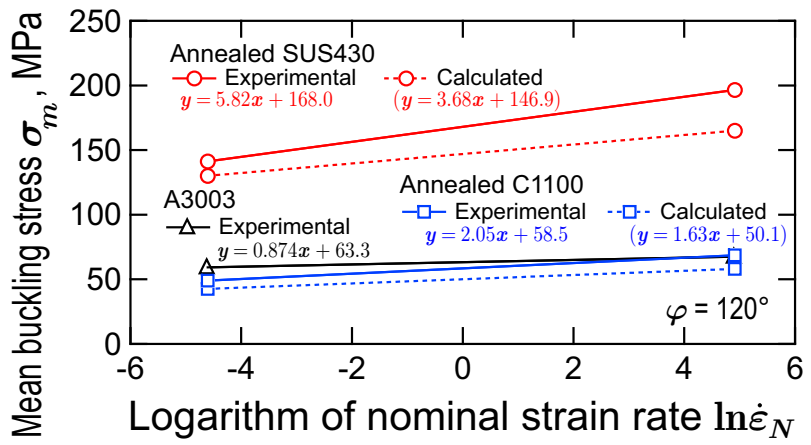

Fig. 8 The strain rate dependence on the mean buckling stress was evaluated for SUS430, C1100 and A3003.

of research have adopted as the axial load per the occupied area of cross section. $\varepsilon_{N}$ was evaluated by dividing $\Delta L$ by the axial length $L$, and the total apparent nominal strain was taken to be 0.5 , corresponding to the displacement of $12.5 \mathrm{~mm}$.

\subsection{Effects of material properties and strain rate}

Figures 7 (a) and (b) show the load $(P)$-displacement $(\Delta L)$ relationships obtained for annealed SUS430 and C1100 honeycombs of $\varphi=120^{\circ}$. In Fig. 7 (a), though the experimental $P-\Delta L$ relationship of SUS430 honeycomb at $V=$ $3.5 \mathrm{~m} / \mathrm{s}$ shows the vibration of high frequency due to the inertial force, at the first peak $P$ increases about $7 \mathrm{kN}$ as the nominal strain rate rises from $1.0 \times 10^{-2} \mathrm{~s}^{-1}$ to $1.4 \times 10^{2} \mathrm{~s}^{-1}$, and at the plateau region increases about $1.5 \mathrm{kN} \cong 19$ $\%)$ as compared with that at $1.0 \times 10^{-2} \mathrm{~s}^{-1}$ due to the strain rate dependence of foil material. The mean buckling load $P_{m}$ increases about $2.0 \mathrm{kN}(\cong 25 \%)$. The numerical simulations reproduce respective $P-\Delta L$ relationships to some extent except low elastic stiffness in the rising part due to including extra elastic deformation of testing machine. In Fig. 7 (b), $P-\Delta L$ relationships of $\mathrm{C} 1100$ also show the enhancement of load due to the strain rate dependence, while those show gentle peaks due to small initial yield stress and large work hardening rate as shown in Fig. 1 (c). On the other hand, Fig. 10 (a) shows $P-\Delta L$ relationships obtained for A3003 honeycombs by experiments and numerical simulations under some different conditions. Experimental curves obtained by low-speed and dynamic crushing tests show good agreement with each other so that the A3003 honeycomb hardly shows the strain rate dependence. The mean buckling stresses $\sigma_{m}$ for dynamic crushing tests $\left(\cong 1.4 \times 10^{2} \mathrm{~s}^{-1}\right)$ of SUS430, C1100 and A3003 honeycombs become 196.2, 68.6 and 66.3 MPa respectively. $\sigma_{m}$ corresponds to $P_{m}$ per the net area of cross section.

Figure 8 shows the enhancement of $\sigma_{m}$ for $\varphi=120^{\circ}$ due to the increase of strain rate. The slope of the approximated line of $\sigma_{m}-\ln \dot{\varepsilon}_{N}$ relationship shows the strain rate sensitivity, and the slopes for SUS430, C1100 and A3003 honeycombs become 5.82, 2.05 and $0.874 \mathrm{MPa}$ respectively in the experiment. $\sigma_{m}$ for A3003 honeycomb shows a little increase in the experiment while its flow stress hardly shows the strain rate dependence. In the numerical simulation, the slopes for SUS430 and C1100 are qualitatively reproduced and become about 3.68 and $1.63 \mathrm{MPa}$ respectively. The slope of experimental $\sigma_{m}-\ln \dot{\varepsilon_{N}}$ relationship is larger than the calculated one. This might come from that the effect of actual resistance elements, such as the friction among cell walls, on $\sigma_{m}$ is larger than that evaluated in the numerical simulation. 

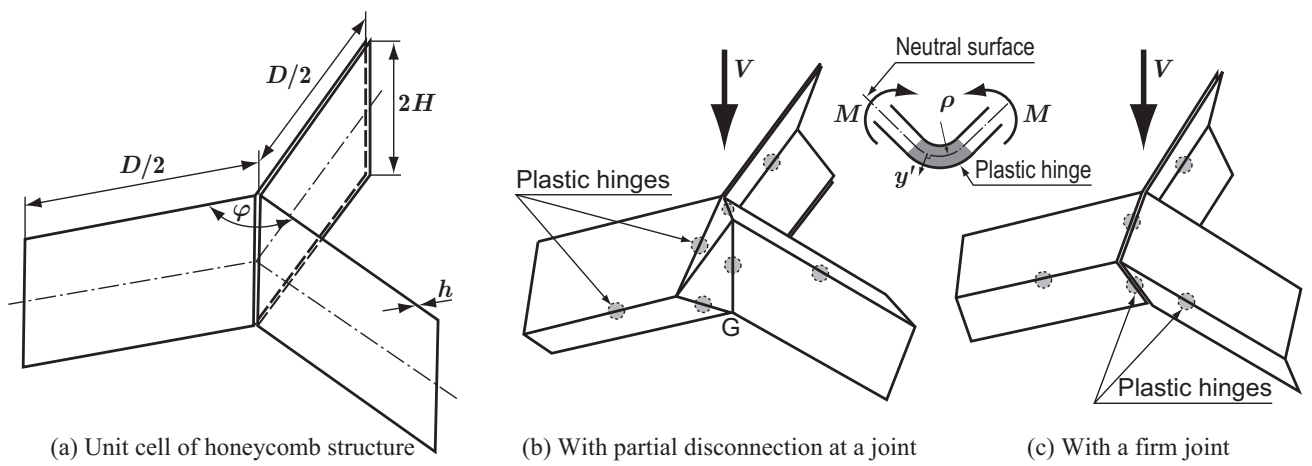

(b) With partial disconnection at a joint

(c) With a firm joint

Fig. 9 Folding mechanism of engineering honeycomb (Wierzbicki, 1983).

The estimation of the strain rate dependence mentioned above is made from the viewpoint in the aggregate, but the substantial resistance in crushing results from the localized progressive plastic buckling at the plastic hinge that forms wrinkles. Then, the substantial strain rate of honeycomb structure should be estimated at the plastic hinge due to bending. Here, to approximately evaluate the substantial strain rate dependence as compared with that of foil material itself, the average plastic strain $\bar{\varepsilon}_{p}^{w}$ across the cell wall thickness and its rate $\dot{\bar{\varepsilon}}_{p}^{w}$ are used. Those were derived by Wierzbicki (1983) for the regular honeycomb on the basis of the rigid-perfectly plastic material approximation as follows:

$$
\begin{aligned}
& \bar{\varepsilon}_{p}^{w}=\frac{2}{h} \int_{0}^{h / 2} \frac{y^{\prime}}{\rho} d y^{\prime}=\frac{h}{4 \rho}, \quad \rho \cong 0.683 \sqrt[3]{h^{2} D} \quad \text { for } D^{\prime}=D \text { and } \varphi=120^{\circ} \\
& \bar{\varepsilon}_{p}^{w}=\frac{d \bar{\varepsilon}_{p}^{w}}{d t} \cong \frac{\bar{\varepsilon}_{p}^{w}}{(2 H / V)}, \quad H \cong 0.821 \sqrt[3]{h D^{2}} \quad \text { for } D^{\prime}=D \text { and } \varphi=120^{\circ}
\end{aligned}
$$

where $\rho$ is the radius of curvature at the plastic hinge, $y^{\prime}$ is the local coordinate perpendicular to the neutral surface of cell wall that is bent at the plastic hinge, and $H$ is a half-length of wrinkle (see Fig. 9). Then, $\bar{\varepsilon}_{p}^{w} \cong 0.1$ and $\dot{\varepsilon}_{p}^{w} \cong 207 \mathrm{~s}^{-1}$ for $h \cong 0.1 \mathrm{~mm}$ and $D=D^{\prime}=3.67 \mathrm{~mm}$. For the experimental data of SUS430 and C1100 foil, shown in Figs. 1 (b) and (c), respectively, $\sigma_{T}$ at $\varepsilon_{p}=0.1$ increases about $32.7 \%(148 \mathrm{MPa})$ and $58.8 \%(68.7 \mathrm{MPa})$ from $1.0 \times 10^{-2} \mathrm{~s}^{-1}$ to $2.1 \times 10^{2}$ $\mathrm{s}^{-1}$ respectively. In the same way, $\sigma_{m}$ increases about 39.2\% (55.4 MPa) and $39.8 \%$ (19.5 MPa) respectively in Fig. 8. Thus, the ratio of increase is not so different, while the increments of $\sigma_{m}$ are only about 37 and $28 \%$ as compared with those of $\sigma_{T}$ at $\varepsilon_{p}=0.1$ for SUS430 and C1100 respectively.

\subsection{Effects of geometry and boundar condition}

Figures 10 (a)-(d) show calculated $P-\Delta L$ relationships obtained for A3003 honeycombs of $\varphi=120,30,60$ and $180^{\circ}$ at $V=3.4 \mathrm{~m} / \mathrm{s}$ respectively. In the following, the boundary condition will be referred to by some term that indicates experimental situation. For example, the normal condition, under which the upper and lower ends of honeycomb core are fully fixed, corresponds to that of crushing honeycomb sandwich panel and will be referred to as "with face plates" if necessary. In the legends of figures, the calculated results under that will be shown only as "Calculated". In Fig. 10 (a) for $\varphi=120^{\circ}$, the calculated results of (i) normal condition, (ii) "without face plates" and (iii) "with face plates and the periodic boundary condition" agree well with each other and also agree well with the experimental results of $V=$ $2.5 \times 10^{-4}, 3.4 \mathrm{~m} / \mathrm{s}$ before the development of densification $(\Delta L \leq 13 \mathrm{~mm})$. In Fig. 10 (d) for $\varphi=180^{\circ}$, the calculated results agree well with each other, while for $\varphi=180^{\circ}$ the experimental peak load in rising part is rather smaller than the calculated peak load that is almost the same value for changing $\varphi$. It seems that the initial imperfection scatters the peak load in the experiment. Furthermore, Fig. 10 (b), (c) for $\varphi=30$ and $60^{\circ}$ show the effects of the boundary condition on the $P-\Delta L$ relationship, and it is found that the calculated results with the boundary conditions (i) and (iii) almost coincide with each other before the densification while the plateau load calculated with the condition (ii) is rather smaller than any other calculated result and is close to the experimental result. In the experiments of $\varphi=30$ and $60^{\circ}$, the partial or total separation of outer foil from the face plate and core was observed so that it can be deduced that the crushing mode in the experiment is close to the condition (ii) in some measure.

Figure 11 shows the effects of $\varphi$ and boundary condition on $\sigma_{m}$. Modeling based on EPZ treatment reduces $\sigma_{m}$ in $\varphi \geq 120^{\circ}$ as compared with that calculated using the seamless model, which is made in one united body and ignores the effect of adhesively-bonded joint on the crushing behavior (LSTC and JSOL, 2007). The contact condition with face 


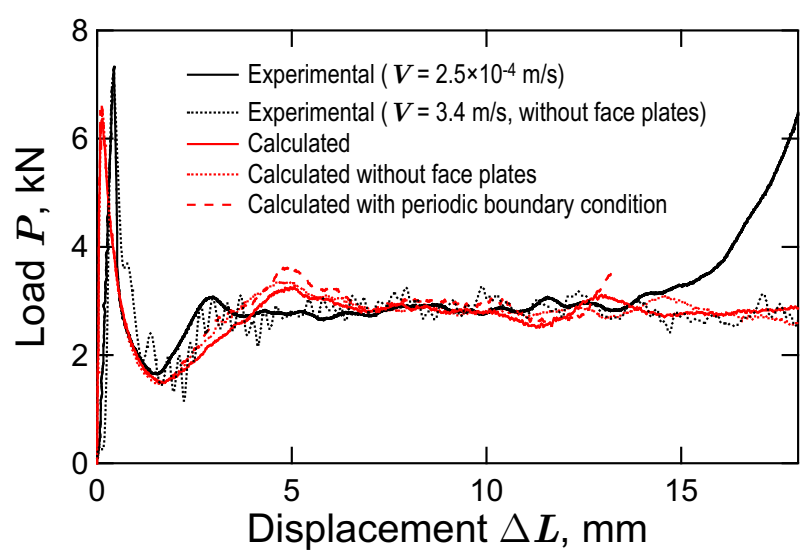

(a) $\varphi=120^{\circ}$

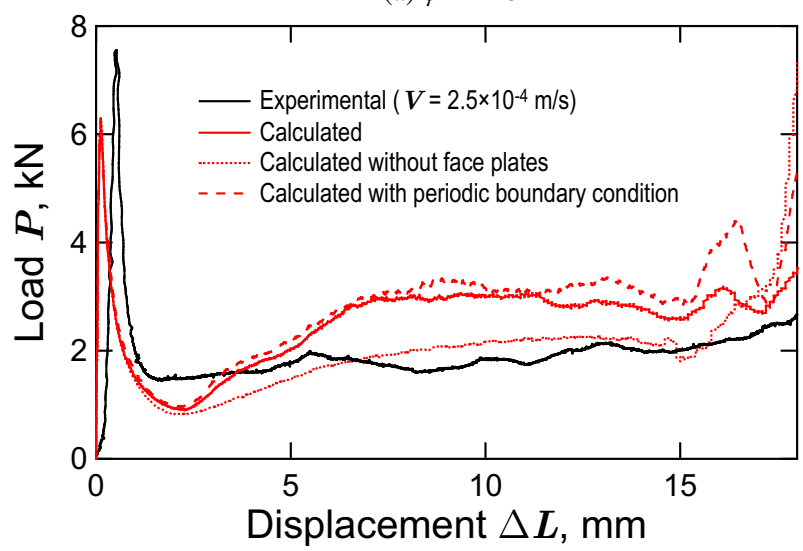

(c) $\varphi=60^{\circ}$

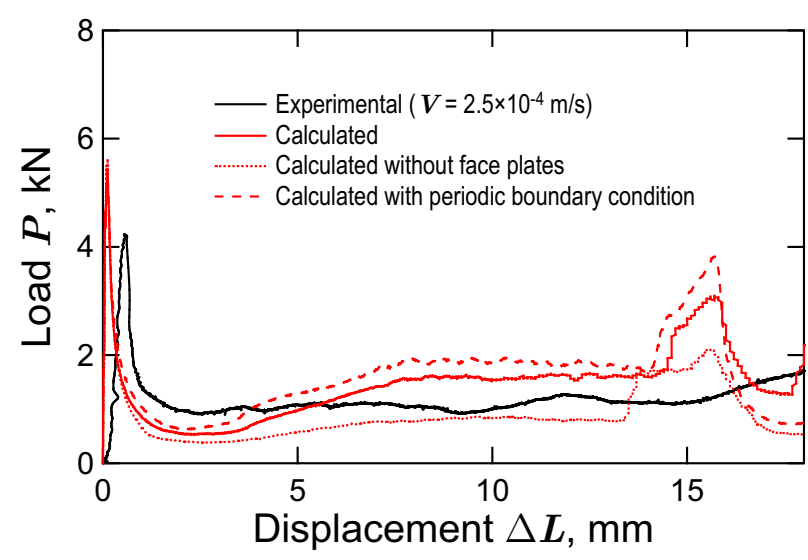

(b) $\varphi=30^{\circ}$

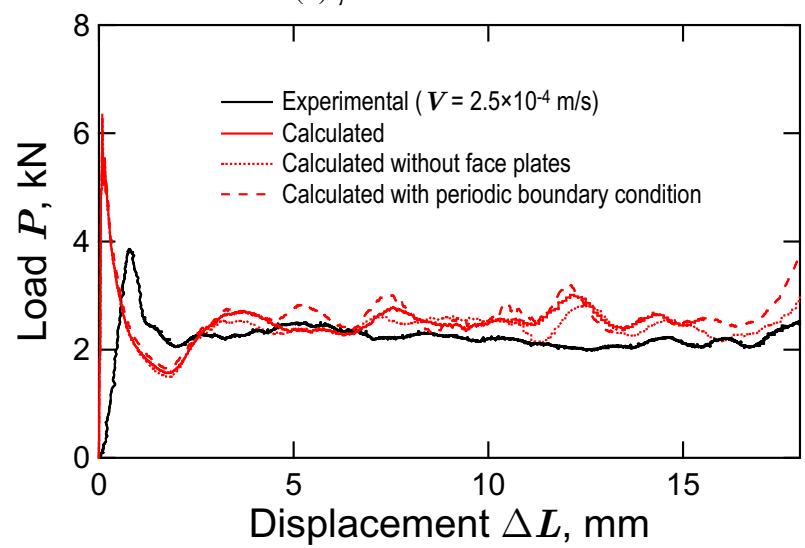

(d) $\varphi=180^{\circ}$

Fig. 10 Load-displacement relationships of A3003 were obtained with varying the branch angle both by the experiment and by the numerical simulation. The experimental results were obtained under low-speed (a)-(d) and impact (a) loading conditions, and the calculated results were obtained under some boundary conditions.

plates greatly affects $\sigma_{m}$ in $\varphi<120^{\circ}$. Adding the periodic boundary condition increases $\sigma_{m}$ a little as compared with that calculated with the normal condition (the core is fixed to the face plates only). The experimental results were obtained by gluing the core to the face plates with epoxy resin adhesive, but in fact the adhesively-bonded joint was partially broken for $\varphi<120^{\circ}$ at relatively early stage due to the effects of free edges and defects such as initial imperfection, adhesive failure and so forth, so that especially $\sigma_{m}$ at $\varphi=60^{\circ}$ is close to the calculated value without face plates. The results without free edges are evaluated by adding the periodic boundary condition, and those imply that in the experiment $\sigma_{m}$ is affected by free edges especially for $\varphi=60^{\circ} . \sigma_{m}$ has a maximum value at $\varphi=120^{\circ}$ except the seamless model.

\subsection{Effect of oblique loading condition}

Figures 12 (a)-(h) show regular A3003 honeycomb core models crushed by the oblique load under the normal boundary condition ("with face plates"). For simplification, the velocity $V$ was applied to the top rigid wall with the crush

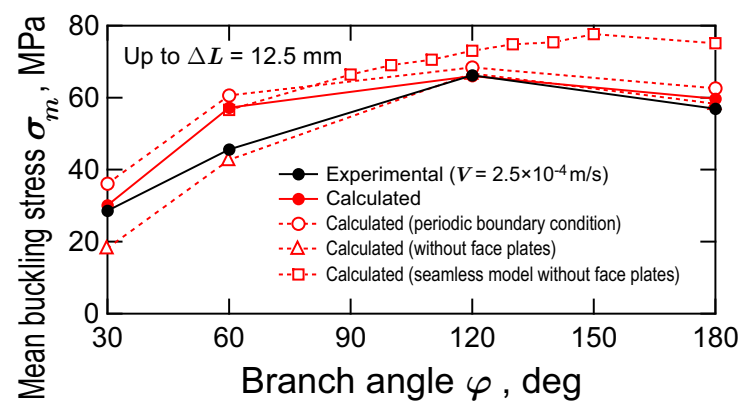

Fig. 11 Effects of boundary conditions on the relationship between mean buckling stress and branch angle (A3003). 


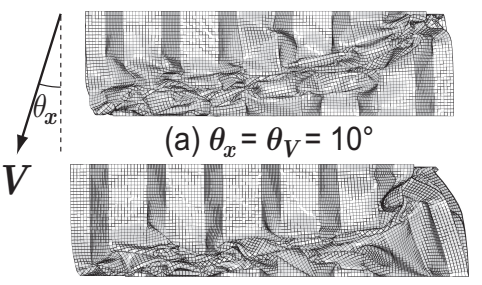

(b) $\theta_{x}=\theta_{V}=20^{\circ}$

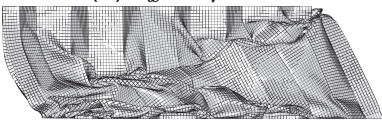

(c) $\theta_{x}=\theta_{V}=30^{\circ}$

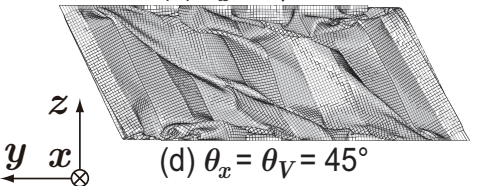

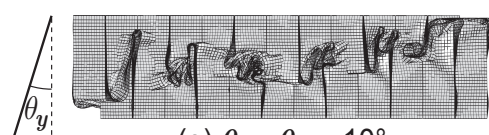

(e) $\theta_{y}=\theta_{V}=10^{\circ}$

$V$

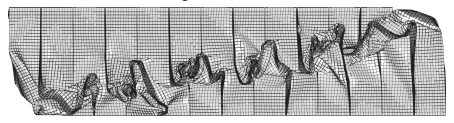

(f) $\theta_{y}=\theta_{V}=20^{\circ}$

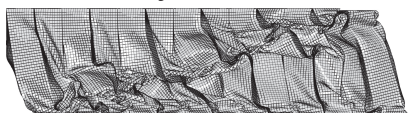

(g) $\theta_{y}=\theta_{V}=30^{\circ}$

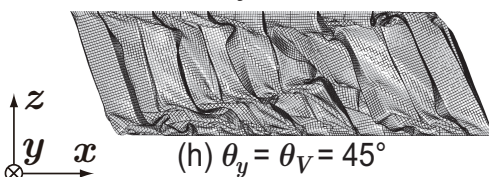

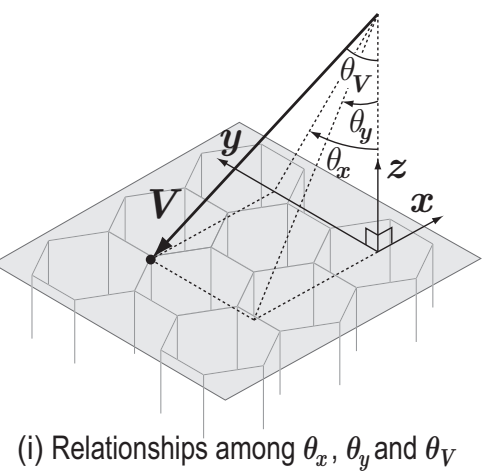

(i) Relationships among $\theta_{x}, \theta_{y}$ and $\theta_{V}$

Fig. 12 Effect of crush angle on the deformation of regular honeycomb core model under the normal boundary condition (A3003, $V=3.4 \mathrm{~m} / \mathrm{s}, t \cong 4.55 \mathrm{~ms}$ ).

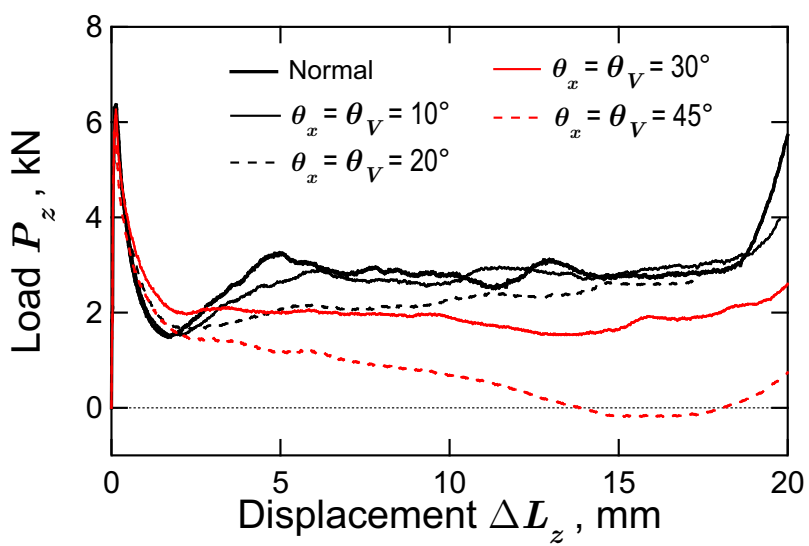

(a) Effect of $\theta_{x}\left(=\theta_{V}, \theta_{y}=0^{\circ}\right)$

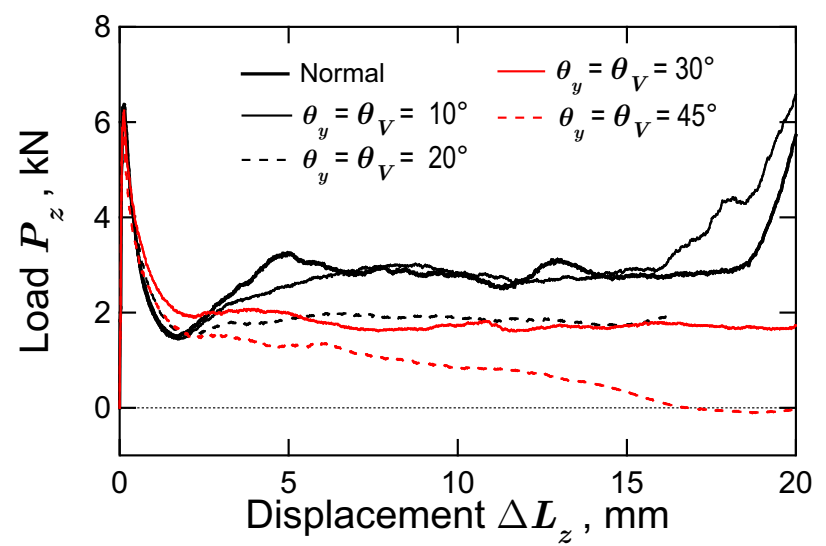

(b) Effect of $\theta_{y}\left(=\theta_{V}, \theta_{x}=0^{\circ}\right)$

Fig. 13 Effect of crush angle on the load-displacement relationship of regular honeycomb sandwich panel model (A3003, $\varphi=120^{\circ}, V=3.4 \mathrm{~m} / \mathrm{s}$ ).

angle $\theta_{\boldsymbol{V}}=\cos ^{-1} \frac{V}{V} \cdot\left(-\boldsymbol{e}_{z}\right), V=|\boldsymbol{V}|=3.4 \mathrm{~m} / \mathrm{s}$, and $\boldsymbol{e}_{z}=(0,0,1)$, and also the angles $\theta_{x}$ around $x$ axis and $\theta_{y}$ around $y$ axis from the normal $(-z)$ direction were introduced to examine the effect of loading direction so that it can be represented as $\boldsymbol{V}=V\left(-\cos \theta_{\boldsymbol{V}} \tan \theta_{y}, \cos \theta_{\boldsymbol{V}} \tan \theta_{x}, \cos \theta_{\boldsymbol{V}}\right)$ as shown in Fig. 12 (i). Here, either of $\theta_{x}$ and $\theta_{y}$ was varied to $10,20,30$ and $45^{\circ}$. In $\theta_{x} \geq 20^{\circ}$ or $\theta_{y} \geq 20^{\circ}$, the axial crushing mode changes to the other mode, which is governed by the bending deformation.

Figure 13 (a) shows the effect of $\theta_{x}$ on the axial load $\left(P_{z}\right)$ - vertical displacement $\left(\Delta L_{z}\right)$ relationship. $P_{z}-\Delta L_{z}$ relationships for $\theta_{x}=0$ and $10^{\circ}$ almost agree with each other, while for $\theta_{x}=20,30$ and $45^{\circ}$ the level of $P_{z}$ decreases with $\theta_{x}$. In the same way, Fig. 13 (b) shows the effect of $\theta_{y}$ on $P_{z}-\Delta L_{z}$ relationship. It seems that the level of $P_{z}$ for $\theta_{y}=20^{\circ}$ decreases more than that for $\theta_{x}=20^{\circ}$, while the difference between those effects is not so considerable.

Figure 14 shows the reduction of the mean buckling load $P_{m}$ with increasing the crush angle $\theta_{V}$ or alternative angles $\theta_{x}$ and $\theta_{y}$. The effect of $\theta_{\boldsymbol{V}}$ on the reduction rate of $P_{m}$ is not so large, however, the reduction rate of $P_{m}$ due to $\theta_{y}$ is larger than that due to $\theta_{x}$ and the mixed condition $\theta_{x}=\theta_{y}$ decreased the largest at $\theta_{\boldsymbol{V}}=45^{\circ}$. In this study, $P_{m}$ at $\theta_{\boldsymbol{V}}=45^{\circ}$ became $30-50 \%$ smaller than that at the vertical impact $\left(\theta_{V}=0^{\circ}\right)$.

\section{Conclusion}

Some metal honeycombs were numerically modeled with taking the effects of adhesively-bonded joint and initial imperfection into account. Then, in order to investigate the effects of branch angle, material properties, strain rate, and crush angle, a series of axial crushing analyses was carried out under low-speed and impact loading conditions. The numerical simulations reproduced well the axial crushing behavior in the experiments for honeycombs of different 


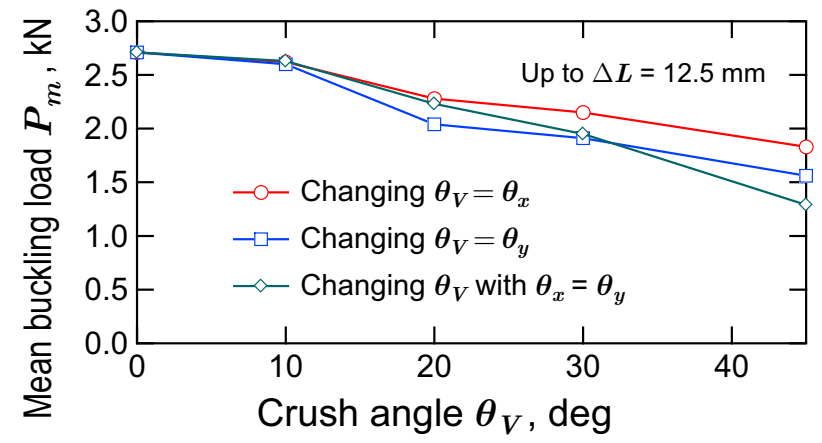

Fig. 14 Effect of crush angle on the mean buckling load of regular honeycomb sandwich panel model (A3003, $\left.\varphi=120^{\circ}, V=3.4 \mathrm{~m} / \mathrm{s}\right)$.

materials and branch angles. The mean buckling stresses of SUS430 and C1100 honeycombs under low-speed and impact loading conditions revealed that the strain rate dependence of the honeycomb structure was relaxed to 30-40\% of that of the raw material. The irregularity in the branch angle $\varphi$ reduced the mean buckling stress; for $\varphi=30^{\circ}$ it was reduced to the smallest value while for $\varphi=180^{\circ}$ it was hardly decreased. The calculated result with the periodic boundary condition suggested that the irregular honeycomb of $6 \times 6$ cells was influenced by free edges especially for $\varphi=60^{\circ}$. Furthermore, the oblique loading under fixed end condition caused the transition of crushing behavior from the axial collapse mode to the bending collapse mode if the crush angle was larger than $20^{\circ}$. This transition of collapse mode greatly decreased the axial load and the energy absorption capacity, and the mean buckling load at the crush angle of $45^{\circ}$ became $30-50 \%$ smaller than that at the vertical impact.

\section{References}

Baker, W.E., Togami, T.C., Weydert, J.C., Static and Dynamic Properties of High-Density Metal Honeycombs, Int. J. Impact Engng., Vol.21, No.3 (1998), pp.149-163.

Chen, D.H., Yoshida, H. and Ozaki, S., Deformation Behaviour for Axial Crushing of Three-Fold Point Corner, Journal of the Japan Society of Mechanical Engineers, Vol.73, No.733 (2007), pp.1037-1044.

Gibson, L.J. and Ashby, M.F., Cellular Solids (Second edition), (1997), Cambridge University Press.

Livermore Software Technology Corp. (Ed.) and JSOL Corporation (Tr.), LS-DYNA Ver.971 User's Manual, Vols.I\&II (2007), JSOL Corporation. (in Japanese)

Ogasawara, N., Shiratori, M., Miyahara, S., Yu, Q. and Miyano, A., Energy Absorption by Honeycomb Structure (2nd Report, Evaluation of Collapsing Stress under Impact Condition), Journal of the Japan Society of Mechanical Engineers, Vol.63, No.616 (1997), pp.2575-2579. (in Japanese)

Tvergaard, V. and Hutchinson, J.W., The Influence of Plasticity on Mixed Mode Interface Toughness, J. Mech. Phys. Solids, Vol.41, No.6 (1993), pp.1119-1135.

Umeda, T., Mimura, K., Hirayama, S., Effects of Geometry and Material Properties on Energy Absorption of Axially Crushing Honeycomb Structure, Proceedings of ATEM'11, CD-ROM (2011), 10 Pages.

Umeda, T., Mimura, K. and Kamigawa, T., Numerical Evaluation of Dynamic Behavior of Axial Crushing Honeycomb Based on Adhesively-Bonded Model, Applied Mechanics and Materials, Vol.566 (2014), pp.605-610.

Wierzbicki, T., Crushing Analysis of Metal Honeycombs, Int. J. Impact Engng., Vol.1, No.2 (1983), pp.157-174.

Wu, E. and Jiang, W.S., Axial Crush of Metallic Honeycombs, Int. J. Impact Engng., Vol.19, Nos.5-6 (1997), pp.439-456.

Yamashita, M. and Gotoh, M., Impact Behavior of Honeycomb Structures with Various Cell Specifications-Numerical Simulation and Experiment, Int. J. Impact Engineering, Vol.32 (2005), pp.618-630.

Yang, Q.D. and Thouless, M.D., Mixed-Mode Fracture Analyses of Plastically-Deforming Adhesive Joints, International Journal of Fracture, Vol.110 (2001), pp.175-187.

Yasui, Y., Dynamic Axial Crushing of Multi-Layer Honeycomb Panels and Impact Tensile Behavior of the Component Members, Int. J. Impact Engng., Vol.24, Nos.6-7 (2000), pp.659-671.

Zhao, H., Gary, G., Crushing Behavior of Aluminium Honeycombs under Impact Loading, Int. J. Impact Engng., Vol.21, No.10 (1998), pp.827-836. 